\title{
Os vinte anos de existência da Paidéia: uma história que vale a pena contar
}

Agosto de 2011. Com a publicação deste fascículo 49 do volume 21, a Paidéia completa 20 anos de existência e celebra seu amadurecimento com um projeto gráfico remodelado e visual arrojado, que a consagraram como uma das grandes revistas de Psicologia do contexto nacional. Com a consolidação de seu prestígio junto à comunidade científica, angariado graças à sua seletiva política de divulgação do conhecimento de qualidade, a Paidéia alcança sua maturidade editorial e reafirma sua vitalidade, pontuada por tantas conquistas.

De fato, temos muito o que comemorar. Coincidindo com a celebração do marco histórico de seus 20 anos, a Paidéia acaba de ser indexada na base Scopus, da Elsevier. Assim, a partir deste número, contamos com mais um indexador de projeção internacional. Para se aferir a relevância dessa conquista, basta mencionar que se trata de uma das mais importantes bases indexadoras do mundo. Com essa importante aquisição, a Paidéia agora está presente em 10 bases indexadoras do Brasil, Estados Unidos, França, México e Espanha.

Completar duas décadas de vida nos obriga a fazer um balanço de onde estamos e para onde pretendemos ir. Nessa oportunidade, gostaríamos sobretudo de dividir os louros advindos da conquista dos frutos do trabalho de gestão editorial iniciado há cerca de quatro anos. Consideramos que esse ensejo festivo deve ser comemorado por todos aqueles que dão sustentação a esse árduo processo de trabalho coletivo que, ao fim e ao cabo, é a seiva que sustenta a vida de uma revista.

A Paidéia nasceu em agosto de 1991, graças à iniciativa de um grupo de docentes do então Departamento de Psicologia e Educação da Faculdade de Filosofia, Ciências e Letras de Ribeirão Preto, da Universidade de São Paulo (FFCLRPUSP). A Profa. Lisete Diniz Ribas Casagrande e o Prof. José Marcelino de Rezende Pinto foram os primeiros editores e, nessa condição, cuidaram de gerir os primeiros passos da revista, que nasceu como Paidéia: Cadernos de Educação.

Já no segundo fascículo, lançado em fevereiro de 1992, a dupla de editores foi reforçada com a entrada das professoras Zélia Maria Mendes Biasoli Alves e Zilma de Moraes Ramos de Oliveira. A Comissão Editorial incorporou nomes de expressão nacional e internacional, alguns vinculados ao Departamento de Psicologia e Educação da FFCLRPUSP: André Jacquemin, Antônio Ruffino Filho, Aureliano Coimbra Filho, Cilene Ribeiro de Sá Leite, Dair Aily Franco de Camargo, José Camilo dos Santos Filho, José Carlos de Araújo Melchior, Mansur Lutfi, Maria Clotilde Rossetti Ferreira, Maria Regina Kawamura, Mauricio Tragtenberg e Sérgio Lorenzato.
Em agosto de 1994, com o lançamento do fascículo 7, a revista passou a denominar-se Paidéia: Cadernos de Psicologia e Educação, refletindo a ampliação do escopo inicialmente delineado. Nesse número tivemos o afastamento da Profa. Zilma da comissão de editores, permanecendo a Profa. Lisete, Prof. José Marcelino e Profa. Zélia.

A partir do fascículo 12/13, de fevereiro/agosto de 1997, a função de Editor ficou sob a responsabilidade do Prof. José Marcelino de Rezende Pinto e da Profa. Zélia Maria Mendes Biasoli Alves, mantendo-se o restante da Comissão Editorial. Com o número 14/15, de fevereiro/agosto de 1998, apenas a Profa. Zélia Maria Mendes Biasoli Alves permaneceu na função de Editora. Com novo foco, a Comissão Editorial foi inteiramente remodelada, passando a ser composta pelos professores Antônio dos Santos Andrade, Eucia Beatriz Lopes Petean, Geraldo Romanelli, Manoel Antônio dos Santos, Marina Rezende Bazon, Noeli Padilha e Reinaldo Furlan, todos da FFCLRP-USP.

Essa situação perdurou até o número 35, de setembro/ outubro de 2006, sendo que, desde o número 21, de 2001, a Profa. Eucia Beatriz Lopes Petean assumira a função de Editor Associado. O fascículo 36, de janeiro/abril de 2007, foi iniciado pela Profa. Zélia e concluído pelo novo editor, Prof. Manoel Antônio dos Santos. A partir desse número a Paidéia passou a contar com os Editores Associados: Profa. Eucia Beatriz Lopes Petean (que se manteve no desempenho dessa função), Prof. Antônio dos Santos Andrade e Profa. Lucy Leal Melo Silva. A Comissão Editorial passou a ser constituída pelos professores Geraldo Romanelli, Marcus Vinicius da Cunha, Marina Rezende Bazon e Reinaldo Furlan

Desde a publicação do fascículo 42, de janeiro/abril de 2009, a denominação Paidéia: Cadernos de Psicologia e Educação foi abreviada para Paidéia, para marcar a inauguração do projeto gráfico totalmente remodelado, com nova capa e layout contemporâneo e arrojado. Editor, Editores Associados e membros da Comissão Editorial permaneceram inalterados. Na composição desta Comissão houve o acréscimo das professoras Kátia de Souza Amorim e Thaís Zerbini.

Com o número 46, de maio/agosto de 2010, a Comissão Editorial incorporou as professoras Edna Maria Maturano e Sônia Regina Loureiro. A função de Editor Associado foi suprimida em prol de uma Comissão Editorial ampliada, que passou a ser constituída pelos professores vinculados ao Programa de Pós-graduação em Psicologia da FFCLRPUSP: Antônio dos Santos Andrade, Edna Maria Maturano, Eucia Beatriz Lopes Petean, Geraldo Romanelli, Kátia de Souza Amorim, Marcus Vinicius da Cunha, Marina Rezende Bazon, Sônia Regina Loureiro, Thaís Zerbini. A partir do 
número 48, de maio/agosto de 2011, deu-se o afastamento do Prof. Marcus Vinicius da Cunha e houve a incorporação, no Corpo Editorial, da Profa. Andréia Schmidt.

O Conselho Editorial, estruturado em função das áreas e dos temas abordados nos artigos submetidos à apreciação da Paidéia, é constituído por nomes expressivos da comunidade acadêmica nacional e internacional.

O processo de indexação da revista teve início em 2000, junto à base LILACS - Literatura Latino-Americana e do Caribe em Ciências da Saúde (Centro Latino-americano y de Caribe de Informaciones em Ciências de la Salud), seguida, em 2002, do Index-Psi Periódicos (Conselho Federal de Psicologia - CFP / Pontifícia Universidade Católica de Campinas - PUCCAMP). Entre 2003 e 2004, a Paidéia foi incluída em três novas bases para indexação: PsycINFO (American Psychological Association - EUA), CLASE - Citas Latinoamericanas en Ciências Sociales y Humanidades (Universidad Nacional Autónoma de México) e PSICODOC (Colégio Oficial de Psicólogos de Madrid / Universidad Complutense de Madrid).

Em 2002, a Paidéia obteve seu credenciamento junto ao SIBI (Sistema Integrado de Bibliotecas da USP), órgão que congrega e avalia, anualmente, os periódicos vinculados à Universidade de São Paulo. Desde aquela data, a revista tem obtido avaliação favorável deste órgão, o que assegura a provisão de recursos financeiros indispensáveis para sua continuidade. O credenciamento junto ao SIBI permitiu dinamizar a produção dos fascículos e obter o aval da Universidade para encaminhar a solicitação da inclusão da Paidéia no sistema SciELO - Scientific Electronic Library Online, no qual a revista foi aceita em 2006 e disponibilizada a partir de 2007.

Em 2010 a Paidéia foi indexada no Ulrich's International Periodicals Directory e Catálogo Latindex. Em 2011, foi aceita nas bases Scopus (Elsevier) e PASCAL (Institut de l'Information Scientifique et Technique - INIST). Assim, atualmente, a revista encontra-se indexada nas seguintes bases: SciELO, Scopus, PsycINFO, PASCAL, LILACS, CLASE, PSICODOC, Ulrich's, Latindex, Index-Psi Periódicos.

Desde 2002, a Paidéia está vinculada à Associação Brasileira de Editores Científicos (ABEC). Também é vinculada à Associação Brasileira de Editores Científicos de Psicologia (ABECiPsi).

Ao repassarmos as duas décadas de divulgação da produção científica, é possível apreender, nos artigos publicados na Paidéia no decorrer desse período, as temáticas e desenhos metodológicos que se tornaram recorrentes e prosperaram no campo científico, assim como aqueles que arrefeceram e perderam força, como é esperado em se tratando de um domínio científico caracterizado pela dispersão tanto teórica como metodológica. Norteados por um olhar histórico, podemos encontrar certos padrões recursivos ou, então, a dispersão de elementos, o que permite captar continuidades e rupturas que conferem inteligibilidade às mudanças operadas nas últimas décadas.
A finalidade precípua de um periódico é acolher e dar visibilidade à produção científica de excelência em sua área de abrangência. $O$ trabalho de manter em funcionamento uma revista é colossal e demanda ações coordenadas de um número significativo de profissionais, especialistas, autores, consultores ad hoc, técnicos especializados, funcionários, bolsistas, colaboradores engajados nas diferentes tarefas que são exigidas, sobretudo quando o periódico existe em dois ambientes, isto é, em sua versão eletrônica e impressa.

Nessa direção, este é o momento de louvar o trabalho vivo daqueles que estão na linha de frente da Paidéia. É hora de agradecermos àqueles que contribuíram para que esse marco pudesse ser alcançado e que tiveram participação ativa no aperfeiçoamento contínuo deste periódico ao longo dos anos. Um projeto editorial bem sucedido como a Paidéia não pode se sustentar sem o empenho de pessoas que arregaçam as mangas e colocam a mão na massa, cuidando da faina diária que faz esse periódico acontecer naquilo que ele tem de mais valoroso: a apreciação do mérito científico dos manuscritos submetidos pelos autores. É preciso reconhecer os esforços e a luta pertinaz da Comissão Editorial para manter a qualidade dos textos publicados pela Paidéia no cenário nacional e internacional. Juntamente com os membros ilustres do Conselho Editorial, constituem um grupo de professores e pesquisadores altamente qualificados, que não medem esforços pessoais para se dedicarem à tarefa de projetar a produção qualificada em Psicologia, buscando mediar o julgamento isento dos pares.

Gostaríamos de destacar o valioso trabalho empreendido pela equipe técnica: na função de Assistente Editorial, Eduardo Name Risk e Érika Arantes de Oliveira-Cardoso; no Apoio Técnico: Gleice Marques Pereira; na Secretaria: Isilda Marisa Faim Matiusso Alves; na diagramação: Raquel Elizandra Ramos; na revisão dos resumos: Arlete Belluzzo e Sofie T. Aversari Martins; na revisão das normas de publicação e referências bibliográficas: as bibliotecárias Maria Cristina Manduca Ferreira e Márcia Regina da Silva. Finalmente, na concepção da nova capa: Murilo Ohswald Máximo.

Do ponto de vista da periodicidade, a Paidéia nasceu como revista semestral e, a partir do número 27 , de janeiro/abril de 2004, tornou-se quadrimestral. Um dos maiores desafios é manter a periodicidade e regularidade da publicação. Nesse sentido, o Programa de Pós-graduação em Psicologia, a Chefia do Departamento de Psicologia e a Diretoria da Faculdade de Filosofia, Ciências e Letras de Ribeirão Preto têm fornecido insumos preciosos, que incluem a manutenção da infra-estrutura material e administrativa necessária para a revista funcionar. O Programa de Apoio às Publicações Científicas Periódicas da USP, por meio de sua Comissão de Credenciamento, como mencionamos anteriormente, tem mantido apoio regular, por meio do aporte dos recursos necessários para custear nossas despesas mais vultosas. Paralelamente, desde 2003, temos sido agraciados com recursos provenientes dos editais a que concorremos, anualmente, do Programa Editorial do Conselho Nacional de 
Desenvolvimento Científico e Tecnológico (CNPq) - Editoração e Publicação de Periódicos Científicos Brasileiros.

Em tempos de acesso universal, imediato e quase ilimitado ao conhecimento produzido pela comunidade científica, o cientista precisa dominar habilidades e competências necessárias não apenas para gerar novos conhecimentos, como também para comunicar, de forma clara e objetiva, o seu produto. É preciso adquirir mestria para compartilhar com os pares o saber consolidado, exercitando habilidades de escrita, precisão e qualidade textual. No cenário nacional, infelizmente ainda não temos uma tradição consolidada na prática de redigir artigos, o que muitas vezes se reflete na precariedade dos textos submetidos às revistas. Também procuramos manter a consciência crítica em relação aos alcances e limites inerentes à produção do conhecimento. Disponibilizamos nosso acervo para acesso livre, colaborando com a disseminação do conhecimento, buscando assegurar a confiabilidade dos conteúdos veiculados. Além disso, temos investido na ampliação das bases indexadoras.

Em relação ao presente número da revista, neste fascículo 49 do volume 21 a seção dedicada aos artigos é aberta com a contribuição internacional: Cor e produção de respostas ao Rorschach: um estudo transcultural, da autoria de Danilo Rodrigues Silva e Ana Sousa Ferreira, da Universidade de Lisboa. O estudo investigou o caráter cultural ou natural do efeito de aumento da produção de respostas ao Rorschach, a partir dos 15-16 anos, por meio da comparação de psicogramas de sujeitos portugueses e moçambicanos. Os resultados evidenciaram que o desenvolvimento da percepção cromática se processa diferentemente nos referidos grupos, o que possivelmente está relacionado a fatores ecológicos, culturais e sociais.

A seguir, o artigo intitulado Motivação para aprender: relação com o desempenho de estudantes, de Rita da Penha Campos Zenorini, Acácia Aparecida Angeli dos Santos e Rebecca de Magalhães Monteiro, da Universidade São Francisco, teve como objetivo comparar as orientações de metas de estudantes com alto e baixo desempenho e identificar diferenças relativas às variáveis sexo e tipo de escola. Foi aplicada a Escala de Avaliação da Motivação para Aprendizagem em um grupo de 110 estudantes de ensino médio. Os dados obtidos estão em consonância com a literatura da área e apontam desdobramentos relevantes para futuras pesquisas.

$\mathrm{O}$ terceiro artigo, Representações sociais, conhecimento científico e fontes de informação sobre aids, assinado por Jean Carlos Natividade, da Universidade Federal do Rio Grande do Sul e Brígido Vizeu Camargo, da Universidade Federal de Santa Catarina, investigou as relações entre a valorização simbólica atribuída a elementos descritores da representação social da aids, o nível de conhecimento científico sobre a temática e suas fontes para obtenção de informações. A amostra foi composta por 150 estudantes do ensino médio que responderam a um questionário. Os resultados do estudo oferecem subsídios importantes para o planejamento de estratégias de atenção à saúde para a população jovem.
O quarto trabalho, intitulado $O$ significado da morte para adolescentes, adultos e idosos, de Caroline Garpelli Barbosa, Lígia Ebner Melchiori e Carmen Maria Bueno Neme, da Universidade Estadual Paulista, abordou como pessoas de diferentes gerações lidam com perdas de entes queridos e concebem sua própria finitude, revelando diferenças nas representações desses temas em cada grupo. As autoras apontaram a necessidade de estudos futuros incluírem, de forma mais sistematizada, variáveis como religião e classe social.

Na sequência, o artigo Percepções de pessoas com baixa visão sobre seu retorno ao mercado de trabalho, de Zelia Zilda Lourenço de Camargo Bittencourt e Ana Maria Ribeiro da Fonseca, da Universidade Estadual de Campinas, investigou as características de um grupo de pessoas com deficiência visual, atendidas em um programa de reabilitação, e suas expectativas de retorno ao mercado de trabalho. As pesquisadoras concluíram que, a despeito das políticas afirmativas para pessoas com necessidades especiais, fatores como baixa escolaridade e parca qualificação profissional ainda dificultam a inclusão dessa população no mercado de trabalho.

Dando continuidade à temática inclusão, o artigo $E d u$ cação inclusiva: análise e intervenção em uma sala de recursos, de Marileide Antunes de Oliveira, da Universidade Federal de São Carlos, e Lúcia Pereira Leite, da Universidade Estadual Paulista, analisou o funcionamento de uma sala de recursos e descreveu uma intervenção realizada com uma professora especialista. Ao investigarem a instituição estudada, o currículo proposto aos alunos, plano de ensino, dentre outras características, as autoras apontaram divergências entre as políticas governamentais para área e as práticas engendradas na escola, que puderam ser atenuadas a partir da intervenção proposta pela pesquisa.

O sétimo artigo, Estrela que vem do Norte: os baianos na umbanda de São Paulo, da autoria de Alice Costa Macedo e José Francisco Miguel Henriques Bairrão, da Universidade de São Paulo, Ribeirão Preto, analisou a categoria espiritual dos baianos que compõem o panteão umbandista do sudeste paulista. A partir da observação participante, realizada em terreiros, e de entrevistas realizadas com médiuns em transe, conduzidas de acordo com o referencial etnopsicológico, os autores apontaram que as narrativas dos baianos revelam justaposições com aspectos da cultura nordestina, sugerindo que há uma transmutação de cenas regionais em metonímias e metáforas, que se recombinam de modo a não haver uma reflexão imediata de tipos socialmente dados.

Na sequência, o artigo Discursos de pais e mães sobre a amizade em famílias com filhos adolescentes, assinado por Márcia Stengel, da Pontifícia Universidade Católica de Minas Gerais, analisa de que modo as transformações na família contemporânea levaram a modificações nas relações enfeixadas por pais, mãe e filhos. Se a tradicional autoridade parental, sobretudo paterna, atualmente admite novas composições, arrefecendo-se em alguns segmentos sociais, observa-se contradição entre os ideais de relação familiar e de 
amizade. Segundo a autora, essa contradição, se por um lado promove o relacionamento paterno-filial, por outro também mascara possíveis conflitos, tanto entre pais e filhos como entre irmãos.

A seguir, o artigo intitulado Repercussão da violência na mulher e suas formas de enfrentamento, de Ana Cláudia Wendt dos Santos e Carmen Leontina Ojeda Ocampo Moré, da Universidade Federal de Santa Catarina, discute um tema candente para as ciências humanas e da saúde, a partir de um estudo exploratório e descritivo. Foram realizadas entrevistas com dez mulheres expostas à situação de violência perpetrada por parceiro íntimo, que prestaram queixa em uma delegacia especializada. As pesquisadoras constataram que essa modalidade de violência repercute na mulher por meio de sintomas como insônia, instabilidade emocional, ideação suicida e isolamento social. Dentre as estratégias de enfrentamento utilizadas para lidar com seus efeitos perturbadores, as autoras destacaram a busca de diálogo com amigos, familiares e colegas de trabalho.

Na sequência, o estudo intitulado Formação do pesquisador: resultados de cursos de pós-graduação em educação, da autoria de Maria Benedita Lima Pardo, da Universidade Federal de Sergipe, e Neucideia Aparecida Silva Colnago, analisou como alunos de dois cursos de mestrado na área de Educação avaliam sua formação em pesquisa na graduação e na pós-graduação.

$\mathrm{O}$ artigo teórico Interfaces entre funções executivas, linguagem e intencionalidade, da autoria de Lauren Tonietto, Gabriela Peretti Wagner, Clarissa Marceli Trentini, Tania Mara Sperb e Maria Alice de Mattos Pimenta Parente, da Universidade Federal do Rio Grande do Sul, abordou o desenvolvimento das funções executivas a partir do ponto de vista biológico, neuropsicológico e cognitivo. O estudo destacou a importância que a linguagem assume nesses processos, enquanto função reguladora da ação intencional. A partir da revisão da literatura e de proposições teóricas, as autoras ressaltaram a importância da inclusão de aspectos do desenvolvimento nos modelos futuros de funções executivas.

O próximo estudo, intitulado Desenvolvimento de capacidades e gestualidade espontânea, assinado por Andrea de Arruda Botelho-Borges e Tânia Maria José Aiello-Vaisberg, da Universidade de São Paulo, São Paulo, analisou, com base no aporte da psicanálise de Winnicott, de que maneira determinada prática clínica pode constituir ambiente facilitador para a integração de competências dissociadas, que passam a ser vividas de modo transicional como experiências criativas. As autoras relatam a experiência de uma oficina dedicada ao desenvolvimento da capacidade e criatividade na produção de textos.

Encerrando a seção destinada aos artigos, o estudo $A$ construção do conceito de resiliência em psicologia: discutindo as origens, da autoria de Juliana Mendanha Brandão, do Centro Universitário de Belo Horizonte, Miguel Mahfoud e Ingrid Faria Gianordoli-Nascimento, da Universidade Federal de Minas Gerais, investigou a história do conceito de resiliência, com sua origem supostamente localizada no campo da resistência dos materiais. A partir de consultas etimológicas em dicionários de diversas línguas, bem como da literatura na área da física e psicologia, os autores questionaram a noção de que o termo resiliência teria sido tomado de empréstimo das ciências físicas, apresentando pesquisadores de ambas as áreas e colocando em discussão o sentido lato com que o termo é empregado.

A seguir, é apresentado o relato de experiência profissional intitulado Criando contextos ecológicos de desenvolvimento e direitos humanos para adolescentes, de Ana Paula Lazzaretti de Souza, Luciana Dutra-Thomé, Eva Diniz Bensaja Dei Schiró, Camila de Aquino Morais e Silvia Helena Koller, da Universidade Federal do Rio Grande do Sul. As autoras descrevem um programa de intervenção para educação em direitos humanos e protagonismo juvenil para adolescentes em situação de vulnerabilidade social, a partir do enfoque da inserção ecológica de Bronfenbrenner.

Na sequência, a comunicação breve, Leitura contextual e o processamento metalinguístico: considerações teóricas, assinada por Márcia Maria Peruzzi Elia da Mota e Silvia Brilhante Guimarães, da Universidade Federal de Juiz de Fora, discute o papel das operações cognitivas e metalinguísticas nas habilidades de leitura.

Por fim, apresenta-se a resenha intitulada Principios teóricos e possibilidades do Rorschach no Brasil, da autoria de Erika Tiemi Kato Okino, da Universidade de São Paulo, Ribeirão Preto. A autora aborda uma coletânea de trabalhos recentemente publicada, que reúne estudos contemporâneos sobre o Teste de Rorschach, intitulada Avanços do Rorschach no Brasil, organizada por Sonia Regina Pasian, professora do Departamento de Psicologia da FFCLRP-USP.

Este fascículo se encerra com as Normas de Publicação. O êxito alcançado nessa trajetória de 20 anos de revista mostra que, mais do que conquistar posição de destaque no cenário nacional, é preciso consolidar os avanços obtidos e caminhar rumo ao reconhecimento internacional. Isso requer convicção, visão prospectiva e compromisso com a difusão científica, além da postura humilde de quem sabe que ainda existe um longo caminho a percorrer para a conquista de patamares mais elevados de excelência editorial.

A hora é de continuar a unir forças e persistir na árdua faina diária.

Manoel Antônio dos Santos Editor 